\title{
Stereotactic MR-guided online adaptive radiation therapy (SMART) for the treatment of liver metastases in oligometastatic patients: initial clinical experience
}

\author{
Gamze Ugurluer ${ }^{1}$, Teuta Zoto Mustafayev ${ }^{1}$, Gorkem Gungor ${ }^{1}$, Banu Atalar ${ }^{1}$, Ufuk Abacioglu ${ }^{1}$ Meric Sengoz , \\ Fulya Agaoglu', Gokhan Demir ${ }^{2}$, Enis Ozyar ${ }^{1}$ \\ ${ }^{1}$ Department of Radiation Oncology, Acibadem Mehmet Ali Aydinlar University School of Medicine, Istanbul, Turkey \\ ${ }^{2}$ Department of Medical Oncology, Acibadem Mehmet Ali Aydinlar University School of Medicine, Istanbul, Turkey
}

Received: November 19, 2020

Revised: December 20, 2020

Correspondence:

Gamze Ugurluer

Department of Radiation Oncology,

Acibadem Mehmet Ali Aydinlar

University School of Medicine,

Istanbul 34450, Turkey

E-mail: gamze.ugurluer@acibadem. edu.tr

ORCID:

https://orcid.org/0000-0002-3357-1753
Purpose: We aimed to present our initial clinical experience on the implementation of a stereotactic MR-guided online adaptive radiation therapy (SMART) for the treatment of liver metastases in oligometastatic disease.

Materials and Methods: Twenty-one patients (24 lesions) with liver metastasis treated with SMART were included in this retrospective study. Step-and-shoot intensity-modulated radiotherapy technique was used with daily plan adaptation. During delivery, real-time imaging was used by acquiring planar magnetic resonance images in sagittal plane for monitoring and gating. Acute and late toxicities were recorded both during treatment and follow-up visits.

Results: The median follow-up time was 11.6 months (range, 2.2 to 24.6 months). The median delivered total dose was $50 \mathrm{~Gy}$ (range, 40 to $60 \mathrm{~Gy}$ ); with a median fraction number of 5 (range, 3 to 8 fractions) and the median fraction dose was $10 \mathrm{~Gy}$ (range, 7.5 to $18 \mathrm{~Gy}$ ). Ninety-three fractions (83.7\%) among 111 fractions were re-optimized. No patients were lost to follow-up and all patients were alive except one at the time of analysis. All of the patients had either complete (80.9\%) or partial (19.1\%) response at irradiated sites. Estimated 1-year overall survival was 93.3\%. Intrahepatic and extrahepatic progression-free survival was $89.7 \%$ and $73.5 \%$ at 1 year, respectively. There was no grade 3 or higher acute or late toxicities experienced during the treatment and follow-up course.

Conclusion: SMART represents a new, noninvasive and effective alternative to current ablative radiotherapy methods for treatment of liver metastases in oligometastatic disease with the advantages of better visualization of soft tissue, real-time tumor tracking and potentially reduced toxicity to organs at risk.

Keywords: Liver, Metastases, Oligometastatic disease, Stereotactic radiation therapy, MRI, Image guidance

\section{Introduction}

Oligometastatic state in cancer patients is frequently encountered in clinical practice, mainly due to improvement in early detection of distant disease sites [1]. Aggressive local as well as systemic treatments have the potential to increase survival in oligometastatic patients [2]. Beside gastrointestinal system malignancies, many other solid tumors spread to the liver, and ultimately $50 \%$ of patients develop liver metastases [3].

For patients with metastatic or primary liver malignancy; surgery,

Copyright (C) 2021 The Korean Society for Radiation Oncology

This is an Open Access article distributed under the terms of the Creative Commons Attribution Non-Commercial License (http://creativecommons.org/licenses/by-nc/4.0/) which permits unrestricted non-commercial use, distribution, and reproduction in any medium, provided the original work is properly cited. 
radioembolization, radiofrequency ablation (RFA), transarterial chemoembolization (TACE), cryotherapy and yttrium-90 microsphere therapy are some of the local treatment options most frequently used. While surgery can be considered as the gold standard approach due to its excellent local control potential, resection is possible in only $20 \%$ of patients. RFA and TACE use is limited mainly due to two reasons: the first being the tumor size and the second proximity to biliary tract, blood vessels or liver dome. Although considered less invasive than surgery, these procedures can also lead to similar complications like bleeding, infection or liver decompensation.

The potential of external beam radiation therapy (RT) as an alternative modality for the treatment of metastatic or primary liver tumors is being explored with increased frequency. The tendency to use high fraction doses (ablative) in a reduced number of fractions in most organ systems is causing a framework shift within the radiation oncology field. Stereotactic body radiation therapy (SBRT) briefly can be defined as highly conformal, ultra hypofractionated, high-dose external RT to extracranial lesions. Adopting this approach to liver tumors can be challenging due to the potential toxicity risk to the uninvolved liver or other nearby radiosensitive organs.

Use of SBRT in the management of liver metastases and hepatic malignancies leads to excellent local control rates [4-7]. Two-year local control as high as $90 \%$ is reported by modern prospective multi-institutional trials involving SBRT for liver metastases [8].

Being one of the most radiosensitive organs, liver has a low tolerance to ablative radiation doses, especially when large volumes are involved [9]. Several dose volume limits ( $>700-800 \mathrm{~cm}^{3}$ of liver tissue to receive $<15-18$ Gy) have been estimated from many clinical studies, confirming that larger volume of irradiated liver leads to increased toxicity [10]. Schefter et al. [11] used a threshold dose of $<15$ Gy for their 3-fraction schedule. This type of normal tissue constraints, called the critical volume model, represented a departure from the traditional Lyman normal tissue complication probability model used by others [12]. Radiation-induced liver disease (RILD) is the major dose limiting concern in the use of SBRT for liver lesions. Therefore, decreasing volumes of treated liver is the main focus of new technologies, since it has the potential to improve outcome both in cirrhotic patients and in those with normal liver function. Magnetic resonance (MR)-guided SBRT, with its ability to better visualize soft tissue and track tumor during treatment, has been shown to be a novel but reproducible and accurate treatment modality for these tumors. This rapidly evolving technology allows for reduced treatment volume and may consequently minimize liver toxicity $[13,14]$.

The continuous and unpredictable motion of abdominal struc- tures combined with respiratory motion results in an inherent positional uncertainty, causing both intra- and inter-fraction changes, an important issue especially for hypofractionated schemes $[15,16]$. The integration of a magnetic resonance imaging (MRI) system and a megavoltage RT system into a single unit has provided the possibility to acquire MRI of the patient in the treatment position. The process of online image-guided radiotherapy (IGRT) has been facilitated by the availability of MRI and MR guidance. In other respects, targeted region can be constantly imaged during the RT delivery without any additional radiation exposure to the patient [17]. Successful liver SBRT relies on accurate IGRT to give sufficient dose to the target volumes while decreasing dose to the organs at risk (OAR) as well as effective motion management during the RT delivery.

The MRIdian Linac system (ViewRay, Oakwood Village, OH, USA) is the first MR linear accelerator (MR-Linac) system with the US Food and Drug Administration clearance offering the ability for real time tracking and adaptive treatment [18]. With the adoption of MR-guided RT (MRgRT) systems for SBRT dosimetric benefits have been published when treating primary or oligometastatic abdominal tumors [19-21]. However, stereotactic MR-guided online adaptive radiation therapy (SMART) is a new technology, and clinical outcomes are unproven and not presented yet. SMART abbreviation was used for the first time in the same context of online adaptive MRgRT by Bohoudi et al. [22].

In this study, we aimed to present our initial clinical experience on the implementation of SMART for the treatment of liver metastases in oligometastatic disease.

\section{Materials and Methods}

This study was approved by Acibadem Mehmet Ali Aydinlar University Institutional Review Board (No. 2020-01/4 dated 09/01/2020). At our department, clinical MRgRT has been performed since September 2018. Between September 2018 and September 2020, 35 patients were treated for liver metastases using SMART. Twenty-one patients having 24 lesions treated for liver metastases in oligometastatic disease were included in this retrospective study. Prior to treatment, written informed consent for treatment delivery was taken from all patients. After the Institutional Review Board approval simulation techniques, treatment data, dosimetric data, toxicity outcomes, follow-up information, and control imagings were retrospectively reviewed. The inclusion criteria of the study were: age older than 18 years, Karnofsky score $>70 \%$, solid tumor (non-hematologic malignancies), oligometastatic disease. Patients having $\leq 5$ metastatic sites were considered as oligometastatic. Patients with extensive disease and treated with a palliative intent were excluded. 
Similar to diagnostic MR scans, all patients were given questionnaires to check for contraindications for MRgRT. All patients underwent computed tomography (CT) simulation with a slice thickness of $1 \mathrm{~mm}$ for dose calculation purposes. The 0.35T MR scans with $1.5 \mathrm{~mm} \times 1.5 \mathrm{~mm} \times 1.5 \mathrm{~mm}$ resolution were acquired based on a balanced free precession technique (True FISP) providing T2/ T1-weighted contrast. Simulation was done in the supine position in the end-exhalation and end-inhalation breath-hold positions in order to determine the most tolerable technique for the patient and the most dosimetrically advantageous technique. MR acquisition took 17-25 seconds with a $45 \mathrm{~cm} \times 45 \mathrm{~cm} \times 24 \mathrm{~cm}$ or 54 $\mathrm{cm} \times 47 \mathrm{~cm} \times 43 \mathrm{~cm}$ field-of-view. Flexible coils, soft bed as well as other equipment such as knee support, headphones and headrest were used in the supine position for simulation and also during treatment. We used gadoxetate for seven patients in order to get a better tumor visualization [23]. If borders of the metastases were difficult to visualize, gadoxetate was injected before simulation and the optimal time for MR acquisition was determined during simulation. For each particular patient, the optimal time founded for better visualization of the metastases at the simulation with gadoxetate was used for fractions.

Pretreatment simulation MR Linac scans were fused with the simulation CT scans and available diagnostic images (MR and/or positron emission tomography-computed tomography [PET-CT]). All target volumes and OAR were contoured on simulation MR dataset by using ViewRay or Eclipse software (Varian, Palo Alto, CA, USA) and subsequently transferred to the treatment planning system of ViewRay. Contoured OAR structures included the liver, small bowel, large bowel, stomach, main vessels, kidneys, heart and lungs, depending the location of treated lesion. The gross tumor volume (GTV) was defined by considering diagnostic imaging. A 3-8 $\mathrm{mm}$ (median, $5 \mathrm{~mm}$ ) isotropic expansion to the GTV was done to create the planning target volume (PTV).

The institutional constraints were used to evaluate the doses to OAR and target volumes (Table 1). Doses to normal tissues met pre-established constraints. All treatments were planned as stepand-shoot intensity-modulated radiotherapy technique. A new high-resolution MR registered with the simulation MR was performed before each fraction. GTV contours were manually adjusted by the attending physician and relevant OAR contours were adjusted to reflect the anatomy of the day. Two treatment plans were generated during each fraction; the baseline re-calculated plan and a re-optimized plan which used the same number and directions of baseline plan beams. The reasons for re-optimization of plans were insufficient target volume coverage ( $>95 \%$ of PTV receives 100\% of the dose), OAR dose violations or both. After patient specific quality assurance, positioning was checked using a cine MR in a
Table 1. Dose constraints used for SMART

\begin{tabular}{ll}
\hline OAR & \multicolumn{1}{c}{ Dose constraints } \\
\hline PTV & $V_{95 \%}$ receives 100\% of $\mathrm{R}$ \\
Liver & Liver volume receiving $<15.7 \mathrm{~Gy}(3 \mathrm{fx})$ \\
& $<18 \mathrm{~Gy}(5 \mathrm{fx}), 700 \mathrm{~mL}$ \\
Stomach & $0.5 \mathrm{~mL}<36 \mathrm{~Gy}$ and $1 \mathrm{~mL}<33 \mathrm{~Gy}(5 \mathrm{fx})$ \\
& $0.5 \mathrm{~mL}<28.9 \mathrm{~Gy}$ and $1 \mathrm{~mL}<26.6 \mathrm{~Gy}(3 \mathrm{fx})$ \\
Duodenum & $0.5 \mathrm{~mL}<36 \mathrm{~Gy}$ and $1 \mathrm{~mL}<33 \mathrm{~Gy}(5 \mathrm{fx})$ \\
& $0.5 \mathrm{~mL}<28.9$ Gy and $1 \mathrm{~mL}<26.6 \mathrm{~Gy}(3 \mathrm{fx})$ \\
Colon & $0.5 \mathrm{~mL}<36$ Gy and $1 \mathrm{~mL}<33 \mathrm{~Gy}(5 \mathrm{fx})$ \\
& $0.5 \mathrm{~mL}<28.9$ Gy and $1 \mathrm{~mL}<26.6 \mathrm{~Gy}(3 \mathrm{fx})$ \\
Small bowel & $0.5 \mathrm{~mL}<36 \mathrm{~Gy}$ and $1 \mathrm{~mL}<33 \mathrm{~Gy}(5 \mathrm{fx})$ \\
& $0.5 \mathrm{~mL}<28.9 \mathrm{~Gy}$ and $1 \mathrm{~mL}<26.6 \mathrm{~Gy}(3 \mathrm{fx})$ \\
Spinal cord & Maximum dose less than $30 \mathrm{~Gy}(5 \mathrm{fx})$ \\
& Maximum dose less than $21.9 \mathrm{~Gy}(3 \mathrm{fx})$ \\
Right kidney & $\mathrm{V}_{12}<10 \%$, mean less than $10 \mathrm{~Gy}$ \\
Left kidney & $\mathrm{V}_{12}<10 \%$, mean less than $10 \mathrm{~Gy}$ \\
Vessels & Maximum dose less than $53 \mathrm{~Gy}(5 \mathrm{fx})$ \\
& $\mathrm{D}_{47}<10 \mathrm{~mL}(5 \mathrm{fx})$ \\
& Maximum dose less than $45 \mathrm{~Gy}(3 \mathrm{fx})$ \\
& $\mathrm{D}_{39}<10 \mathrm{~mL}$ (3 fx)
\end{tabular}

SMART, stereotactic MR-guided online adaptive radiation therapy; OAR,

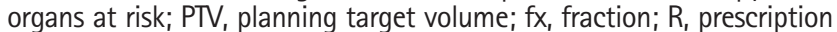
dose.

single sagittal plane and then RT delivery started. During delivery, real-time imaging was used by acquiring planar MR images in one sagittal plane for intra-fraction monitoring and gating. As we could directly visualize the tumor, no other motion-management systems or immobilization devices were used. The SBRT consisted of a median fraction number of 5 (range, 3 to 8 fractions) and majority of patients were treated every other day. The time for workflow steps was documented for each patient; set up, low/high-resolution scanning, contouring, dose prediction and re-optimization with online quality assurance $(Q A)$, real-target cine MR imaging, beam delivery and unexpected disruption of treatment. A more detailed time analysis according to anatomical regions of MRgRT workflow steps conducted by the relevant staff was published previously [24].

Premedication was done with oral antiemetic before each fraction. Acute and late toxicities (nausea, vomiting, inappetence, increased bowel movement, obstipation, meteorism, skin reaction, fatigue, fever, abdominal pain, pneumonitis, cholecystitis, rib fracture) were graded and recorded in patients both during treatment and follow-up visits by treating physician. Patients were followed every three months with PET-CT or MRI.

No patients were lost to follow-up and all but one patient were alive at the time of this analysis. Time intervals were calculated from the first day of SMART. Survival analysis was done according to the Kaplan-Meier method. Overall survival (OS) was calculated 
from the first date of SMART to the day of last follow-up or death. Progression-free survival (PFS) was calculated for both intrahepatic failure and extrahepatic failure. A p-value below 0.05 was accepted as significant. Data analysis was performed using SPSS version 23.0 (IBM, Armonk, NY, USA).

\section{Results}

Between September 2018 and September 2020, 21 oligometastatic patients (24 lesions) from different primaries were treated with SMART for liver metastases. The baseline patient and treatment characteristics are summarized in Table 2. The median follow-up time from first day of SMART was 11.6 months (range, 2.2 to 24.6 months). The median age was 62 years (range, 42 to 86 years). Sixty-two percent of patients were female and 38\% were male. Primary diagnoses were cancer of the colorectum (8 patients, 38\%), breast (4 patients, 19\%), lung (3 patients, 14.3\%), stomach (3 patients, 14.3\%), anal canal (1 patient, 4.8\%), pancreas (1 patient, $4.8 \%$ ) and ovary (1 patient, 4.8\%). All of the patients were treated for their primaries with curative intent, either with surgery or chemotherapy. One of the patients had brain metastases treated with SRS, one had one peritoneal implant treated with MR Linac simultaneously with liver metastases and one patient had lung metastases treated with Linac based SBRT.

All of the initial plans met treatment and OAR constraints. All patients completed planned treatment and required adaptive planning for $\geq 1$ fraction. Ninety-three fractions (83.7\%) among 111 fractions were re-optimized. The reasons for re-optimization of plans were insufficient target volume coverage (61\%), OAR dose violations (0.3\%), both (20\%), and others (2.4\%). Online OA was done for all adapted treatment plans. On average, the median duration of a SMART fraction was approximately 43.0 minutes (range for all patients, 21.0 to 100.0 minutes). The median beam-on treatment delivery time was 19.0 minutes. The mean time for workflow steps was as follows: set up $5.6 \pm 1.7$ minutes, low resolution scanning $1.6 \pm 0.9$ minutes, high resolution scanning $2.6 \pm 2.1$ minutes, online contouring $6.9 \pm 4.8$ minutes, re-optimization with online $Q A 4.1 \pm 2.7$ minutes, real-target $3.6 \pm 6.1$ minutes, and beam delivery with gating $20.3 \pm 8.0$ minutes.

Median GTV volume was $7.1 \mathrm{~mL}$ (range, 1.0 to $24.7 \mathrm{~mL}$ ) and median PTV volume was $14.1 \mathrm{~mL}$ (range, 2.9 to $38.6 \mathrm{~mL}$ ). Intravenous gadoxetate was used for seven patients (Fig. 1). Median PTV volume that was visualized by gadoxetate was $13.7 \mathrm{~mL}$ (median GTV, $7.1 \mathrm{~mL}$ ) and for the lesions contoured without gadoxetate the median PTV volume was $14.5 \mathrm{~mL}$ (median GTV, $7 \mathrm{~mL}$ ), the difference was not significant statistically ( $p>0.05$ ). The median liver volume was $1,296.1 \mathrm{~mL}$ (range, 927.5 to $2,131.0 \mathrm{~mL}$ ). The median of mean
Table 2. Patients' and treatment characteristics

\begin{tabular}{|c|c|}
\hline Characteristic & Value \\
\hline \multicolumn{2}{|l|}{ Sex } \\
\hline Male & $8(38)$ \\
\hline Female & $13(62)$ \\
\hline Age at treatment (yr) & $62(42-86)$ \\
\hline Follow-up (mo) & $11.6(2.2-23.6)$ \\
\hline \multicolumn{2}{|l|}{ Primary tumor } \\
\hline Colorectal & $8(38)$ \\
\hline Breast & $4(19)$ \\
\hline Lung & $3(14.3)$ \\
\hline Stomach & $3(14.3)$ \\
\hline Anal canal & $1(4.8)$ \\
\hline Pancreas & $1(4.8)$ \\
\hline Ovary & $1(4.8)$ \\
\hline Total number of lesions treated & 24 \\
\hline Total PTV dose (Gy) & $50(40-60)$ \\
\hline Fractions & $5(3-8)$ \\
\hline Fraction dose & $10(7.5-18)$ \\
\hline Adapted fractions & $93(83.7)$ \\
\hline $\mathrm{EQD}_{2}$ for $\alpha / \beta=10(\mathrm{~Gy})$ & $83.3(60-126)$ \\
\hline $\mathrm{BED}_{10}$ (Gy) & $100(72.0-151.2)$ \\
\hline $\mathrm{BED}_{3}(\mathrm{~Gy})$ & $216.7(146.7-378)$ \\
\hline GTV $(\mathrm{mL})$ & $7.1(1.0-24.7)$ \\
\hline PTV (mL) & $14.1(2.9-38.6)$ \\
\hline Overall treatment time for each fraction (min) & $43.0(21-100)$ \\
\hline
\end{tabular}

Values are presented as number (\%) or median (range).

PTV, planning target volume; $\mathrm{EQD}_{2}, 2$ Gy equivalent dose; $\mathrm{BED}_{10}$, biologically equivalent doses with $\alpha / \beta=10 ; B_{3}$, biologically equivalent doses with $\alpha / \beta=3$; GTV, gross tumor volume.
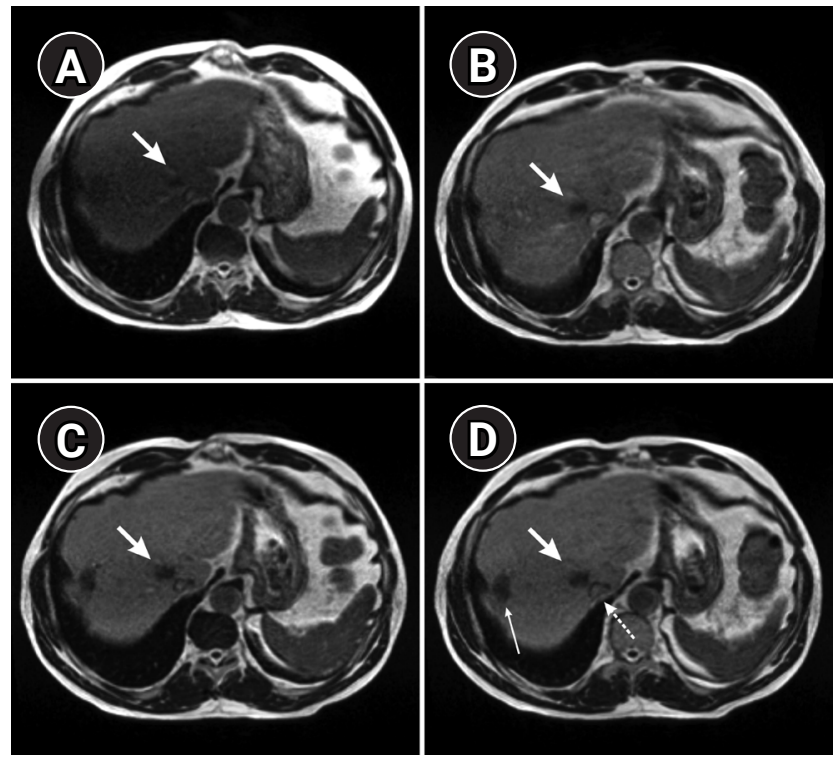

Fig. 1. Axial MRIdian high-resolution breath-hold slices of a patient after intravenous gadoxetate administration shows whitening of liver parenchyma and better visualization of metastatic lesion (thick arrow), previous radiofrequency site (thin arrow), and vena cava inferior (dotted arrow) at (A) baseline, (B) 10 minutes, (C) 17 minutes, and (D) 27 minutes. 
liver doses was $5.1 \mathrm{~Gy}$ (range, 1.4 to $8.9 \mathrm{~Gy}$ ). The distribution of metastatic lesions according to liver segments were as follows: segment IV (6 lesions), VII (6 lesions), V (4 lesions), VIII (4 lesions), VI (2 lesions), and I and III (1 lesion in each). Eighteen patients were treated at end-inhalation breath hold, two patients were treated at end-exhalation breath hold and one patient was treated with free breathing with gating due to non-compliance with the technique.

The median delivered total dose was $50 \mathrm{~Gy}$ (range, 40 to $60 \mathrm{~Gy}$ ); with a median fraction number of 5 (range, 3 to 8 fractions) and the median fraction dose was $10 \mathrm{~Gy}$ (range, 7.5 to $18 \mathrm{~Gy}$ ). The median $\mathrm{EQD}_{2}$ (2 Gy equivalent dose) was $83.3 \mathrm{~Gy}$ (range, 60 to 126 Gy) for $\alpha / \beta=10$. The median $\mathrm{BED}_{10}$ and $\mathrm{BED}_{3}$ (biologically equivalent doses with $\alpha / \beta=10$ and 3) were $100 \mathrm{~Gy}$ (range, 72.0 to 151.2 Gy) and $216.7 \mathrm{~Gy}$ (range, 146.7 to $378 \mathrm{~Gy}$ ), respectively. Eighteen $(75 \%)$ out of 24 lesions were treated with BED $_{10}$ doses of $\geq 100 \mathrm{~Gy}$.

Overall mean survival was 23.4 months, median survival was not reached at the time of this analysis. Estimated 1-year OS was 93.3\% (Fig. 2A). Only one patient was dead at the time of analysis. Progression was defined as intrahepatic and extrahepatic for this cohort of oligometastatic patients. Response evaluation was made by PET-CT in 17 patients (80.9\%) and with contrasted upper abdominal diagnostic MRI in four patients. Although all of the patients had either complete (17 patients, $80.9 \%$ ) or partial (4 patients, 19.1\%) response at irradiated sites, new hepatic metastases were accepted as intrahepatic progression. Intrahepatic PFS was $89.7 \%$ and $64.6 \%$ by Kaplan-Meier analysis at 1 year and 2 years, respectively (Fig. 2B). Extrahepatic progression was recorded as lung metastases in three patients, bone metastases in two patients, brain metastases in one patient, skin metastases in one patient, and supraclavicular lymph node metastases in one patient. While all the patients had various systemic treatments, lung metastasis, bone and brain metastases were treated with SBRT. Extrahepatic PFS was $73.5 \%$ and $42.0 \%$ by Kaplan-Meier analysis at 1 year and 2 years, respectively (Fig. 2 C).

All treatments were very well tolerated. Patients were followed for the acute side effects during treatment and late toxicities after treatment and the Common Terminology Criteria for Adverse Events (CTCAE) version 5.0 was used to record toxicities. Grading was reported as the maximum of any symptom. The most common radiation induced acute toxicities were grade 1 fatigue (mild fatigue over baseline) and nausea (loss of appetite without alteration in eating habits). With regard to acute and late toxicities, there were no grade 3 or higher toxicities experienced during the treatment and follow-up course.

\section{Discussion and Conclusion}

We have presented our clinical experience for the treatment of liver metastases in oligometastatic patients using SMART. Oligometastases term was coined in 1995 by Hellman and Weichselbaum [25] and was defined as "an intermediate state ( $\leq 5$ metastases) between limited primary and polymetastatic cancers in which curative strategies may be effective if all lesions are eradicated" [1]. A randomized phase II international multi-institutional study SABR-COMET (stereotactic ablative radiotherapy for comprehensive treatment of oligometastatic tumors) enrolled patients $<5$ sites of metastatic disease from any primary site (a maximum of three metastases in any one organ was allowed) and randomization was to standard of care or standard of care plus SBRT to all disease sites [26]. In SABR-COMET, 16 among 127 lesions in 66 patients of $\mathrm{SABR}$ group were liver metastases and dose for treatment of liver metastases was 45-60 Gy in 3-8 fractions. The use of SABR result-
(A)

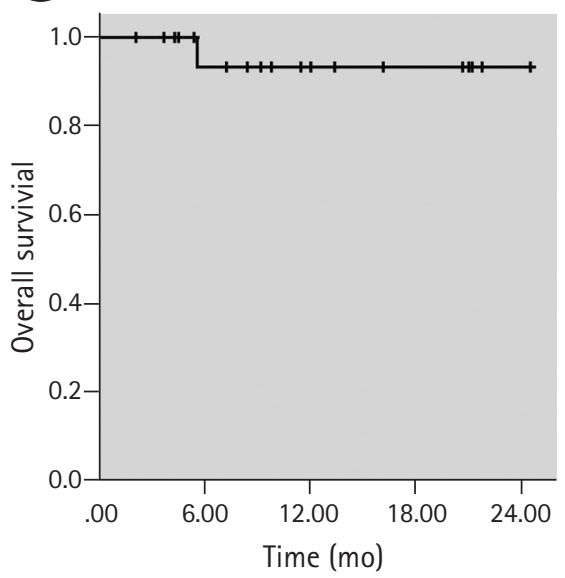

B

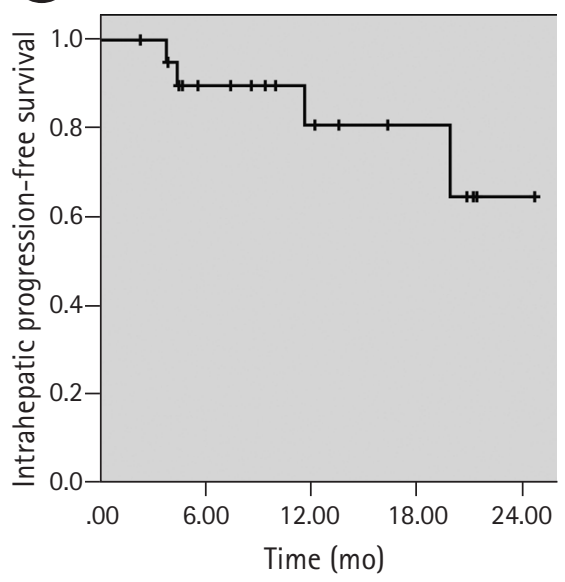

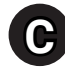

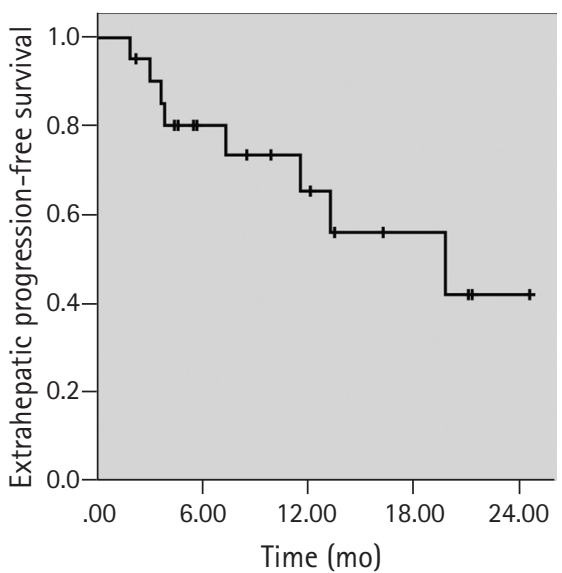

Fig. 2. Kaplan-Meier curves of (A) overall survival, (B) intrahepatic progression-free survival, and (C) extrahepatic progression-free survival. 
ed in a significant improvement in OS (13 months) and the PFS was doubled.

Inter- and intra-fractional motion of the lesion and OAR cause many uncertainties and effects precise delivery of RT to liver lesions. Since most of the radiotherapy machines use kV images or conebeam CT for IGRT, their soft tissue visualization is not enough to track a liver metastasis. Therefore, almost all of the systems other than MR-Linac would need fiducial implementation to track tumors within the soft tissue as well as liver. And it is obvious that fiducial implementation is an invasive procedure with the risk of infection and also additional cost for the patient. MRgRT is the most feasible modality which has the ability of directly imaging liver tumors and OAR altogether and tracking target structure(s) in real time during delivery of RT. Tumors residing in the liver often have highly mobile and radiosensitive OAR in the immediate vicinity such as the bowel, duodenum and stomach thus requiring accurate and continuous imaging. Small changes in these mobile structures can result in violations of dose constraints to OAR, causing increased risk of potential toxicities. A few published series with limited number of patients now exist reporting the efficacy and utility of MRgRT for liver tumors [20,21,27,28].

There are two articles published by Henke et al. [20,21] that evaluated the benefits of adaptive MRgRT for upper abdomen malignancies and one of their studies included patients with liver lesions. In their phase I study, 50 Gy in 5 fractions were evaluated for the treatment of metastatic or unresectable abdominal tumors and there were five patients with metastatic liver tumors. Although all of the dose constraints were met on initial RT planning, due to changes in the anatomy, when this plan was applied to the anatomy of the day, dose constraint violations were identified in nearly $70 \%$ of fractions [20]. Overall, adaptation was performed in 87 (90\%) out of 97 fractions. Ninety-three fractions (83.7\%) among 111 fractions have been re-optimized in our study, as well.

In a retrospective study across three separate institutions 18 patients with liver metastasis were treated with MRgRT, their data detailed the experience using this technology to treat liver tumors with SBRT [27]. This study was characterized by a wide range of prescription dose and volumes. No grade 4-5 toxicities were noticed, only two patients had grade 3 gastrointestinal toxicity. Local control after a median follow-up of 21 months was 80.4\%. Median liver dose in this cohort was $12.7 \mathrm{~Gy}$ (range, 3.2 to $21.9 \mathrm{~Gy}$ ) which was $5.1 \mathrm{~Gy}$ (range, 1.4 to $8.9 \mathrm{~Gy}$ ) in our study. But the patients were treated with a cobalt-based system and moreover median PTV volume was $98.2 \mathrm{~mL}$ (range, 13 to $2,034 \mathrm{~mL}$ ) compared to our patients with a median PTV volume of $14 \mathrm{~mL}$ (range, 2.9 to $38.6 \mathrm{~mL}$ ). One of the patients with grade 3 gastrointestinal toxicity had a large PTV size (>250 mL) and mean liver dose of 21.9 Gy (liv-
er-GTV).

In a study by Padgett et al. [28], online adaptive MR-guided SBRT was compared with non-adaptive SBRT in 10 liver cancer patients and daily re-optimization resulted in better coverage of target volumes and sparing of OAR. PTV dose escalation can be achieved by MR-guided SBRT with OAR sparing as compared to non-adaptive treatment modalities.

The MR-Linac is a new technology with unverified clinical outcomes. Real-time MR images are used both for patient alignment as well as during RT for tracking. Radiation treatment and tracking based on MR images confer clear advantages; such as target and OAR delineation improvement with the better soft tissue visualization, better tracking with smaller margins, elimination or minimization of surrogates [10]. There is no imaging dose delivered to the patients and tumor tracking is consistent with real-time visualization of both the tumor and nearby OAR. MRgRT uses no fiducials that requires an invasive procedure for placement, which might migrate before or during fractions, and the changing positions of OAR cannot be visualized.

It is very well known that SBRT for liver metastases is a safe treatment with a very low risk of clinically important liver toxicity. This is mainly due to lack of important organs around the liver parenchyma. Bleeding and infection are unexpected side effects of SBRT in the literature. Main toxicity with liver SBRT can be radiation induced liver disease. However, even this is a quite rare side effect if universally accepted rules were followed during liver SBRT. Results of the multi-Institutional Phase I/II Trial of Stereotactic Body Radiation Therapy for Liver Metastases by Rusthoven et al. [8], which was published in 2009, revealed that, none of the patients who received post-SBRT systemic therapy experienced high-grade radiation toxicity, and none who later received bevacizumab experienced bleeding or thrombotic complications. There have been no instances of grade 4 or 5 toxicity. None of the seven patients who died before becoming assessable for local control had any evidence of treatment-related toxicity. Only, one instance of grade 3 soft tissue toxicity was observed. In a study by Barry et al. [29], it is reported that SBRT was extremely well tolerated, with a very low risk of clinically important toxicity (3/81), resulting in grade 3 nausea, vomiting, or gastritis in 3 individual patients within 1 month of treatment, all resolving by 3 months.

There was no grade 2 or higher classic or nonclassical RILD toxicity recorded within 3 months after SBRT. One patient developed grade 4 platelet toxicity at 3 months after SBRT that was attributable to taking herbal remedies requiring a splenectomy. Authors concluded that SBRT for liver metastases is a safe treatment with a very low risk of clinically important liver toxicity. The nonclinical grade 1 or 2 elevation of transaminases seen in the majority of pa- 
tients (61\%) and a reduction in albumin levels (28\%) occurring within 3 months were more likely in patients with a higher effective liver volume and higher doses to the spared liver.

Moreover, we used gadoxetate which is a paramagnetic contrast agent for seven patients for better tumor visualization during each fraction of the treatment. Gadoxetate is metabolized partially by hepatocytes and during the hepatobiliary phase distributes progressively into the hepatocytes and bile ducts, continuous visualization can be achieved, both primary liver tumors such as hepatocellular carcinoma and cholangiocarcinoma as well as metastases can be visualized [23]. Gadoxetate can effectively be utilized for patients with liver metastases during MRI-guided SBRT aiding tumor localization, reducing PTV margins, tumor tracking, and treatment gating. Gadoxetate for liver MRI has an excellent safety profile and in postmarketing surveillance database, only 0.026\% over 2.2 million patient exposures resulted in any adverse event, and in patients with impaired hepatic or renal function there was not any increased incidence of side effects [30]. However, this data mainly comes from diagnostic imaging and toxicity profile of consecutive use of it during treatment is not known. Thus, we should be cautious while using SMART. Although we did not use gadoxetate for follow up in our patients there are studies evaluating its use for follow up in the literature. Excellent local tumor control has been achieved with SBRT, but the targeting accuracy in real-life practice remains poorly understood. In a study by Jung et al. [31], they proposed an in vivo assessment of targeting accuracy using hepatic parenchymal changes observed on gadolinium ethoxybenzyl diethylenetriaminepentaacetic acid (Gd-EOBDTPA) enhanced MR images and applied this method to investigate the "real-life" targeting accuracy of image-guided SBRT. They concluded that the hepatic parenchymal changes observed on Gd-EOB-DTPA-enhanced MR images can be used to assess the targeting accuracy after SBRT for HCC. In order to evaluate the relationship between the dose to the liver parenchyma and focal liver reaction (FLR) after SABR, a novel method using a three-dimensional dose distribution and change in signal intensity of Gd-EOB-DTPA-enhanced MRI hepatobiliary phase images was suggested. This method was capable of providing a quantitative evaluation of the relationship between dose and intensity changes on follow-up MR, as well as determining individual dose for developing FLR [32].

To our knowledge, our series have the largest number of patients with liver metastases in oligometastatic disease treated with SMART in the literature. Our study has following limitations; retrospective nature, short follow up time, heterogenous group of primary diagnosis and use of different treatment doses. Strengths of our study are; beside the largest reported number of patients treated with SMART, staging and response evaluation of most of our patients were made using PET-CT without late radiation induced liver toxicity at last follow-up.

In conclusion, SMART represents a new, non-invasive and efficacious alternative to current modalities for treatment of liver metastases. It has the potential to change the management in many patients unsuitable for other treatment approaches. This technology has some advantages when compared to other radiotherapy techniques as well, due to its better visualization of soft tissue, real-time tumor tracking without invasive placement of fiducial markers and potentially reduced toxicity to OAR. Longer follow-up and prospective randomized studies which compare this technique to other modalities are warranted.

\section{Conflict of Interest}

No potential conflict of interest relevant to this article was reported.

\section{References}

1. Weichselbaum RR, Hellman S. Oligometastases revisited. Nat Rev Clin Oncol 2011;8:378-82.

2. Alongi F, Arcangeli S, Filippi AR, Ricardi U, Scorsetti M. Review and uses of stereotactic body radiation therapy for oligometastases. Oncologist 2012;17:1100-7.

3. Ananthakrishnan A, Gogineni V, Saeian K. Epidemiology of primary and secondary liver cancers. Semin Intervent Radiol 2006; 23:47-63.

4. Andratschke NH, Nieder C, Heppt F, Molls M, Zimmermann F. Stereotactic radiation therapy for liver metastases: factors affecting local control and survival. Radiat Oncol 2015;10:69.

5. Goodman BD, Mannina EM, Althouse SK, Maluccio MA, Cardenes HR. Long-term safety and efficacy of stereotactic body radiation therapy for hepatic oligometastases. Pract Radiat Oncol 2016; 6:86-95.

6. Joo JH, Park JH, Kim JC, et al. Local control outcomes using stereotactic body radiation therapy for liver metastases from colorectal cancer. Int J Radiat Oncol Biol Phys 2017;99:876-83.

7. Zeng ZC, Fan J, Tang ZY, et al. A comparison of treatment combinations with and without radiotherapy for hepatocellular carcinoma with portal vein and/or inferior vena cava tumor thrombus. Int J Radiat Oncol Biol Phys 2005;61:432-43.

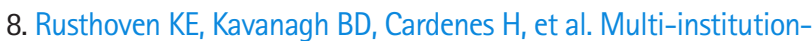
al phase I/II trial of stereotactic body radiation therapy for liver metastases. J Clin Oncol 2009;27:1572-8.

9. Pan CC, Kavanagh BD, Dawson LA, et al. Radiation-associated liver injury. Int J Radiat Oncol Biol Phys 2010;76:S94-100. 
10. Witt JS, Rosenberg SA, Bassetti MF. MRI-guided adaptive radiotherapy for liver tumours: visualising the future. Lancet Oncol 2020;21:e74-82.

11. Schefter TE, Kavanagh BD, Timmerman RD, Cardenes HR, Baron A, Gaspar LE. A phase I trial of stereotactic body radiation therapy (SBRT) for liver metastases. Int J Radiat Oncol Biol Phy 2005;62: 1371-8.

12. Dawson LA, Normolle D, Balter JM, McGinn CJ, Lawrence TS, Ten Haken RK. Analysis of radiation-induced liver disease using the Lyman NTCP model. Int J Radiat Oncol Biol Phys 2002;53:81021.

13. van de Lindt $T$, Sonke JJ, Nowee $M$, et al. A self-sorting coronal 4D-MRI method for daily image guidance of liver lesions on an MR-LINAC. Int J Radiat Oncol Biol Phys 2018;102:875-84.

14. Kishan AU, Cao M, Wang PC, et al. Feasibility of magnetic resonance imaging-guided liver stereotactic body radiation therapy: a comparison between modulated tri-cobalt-60 teletherapy and linear accelerator-based intensity modulated radiation therapy. Pract Radiat Oncol 2015;5:330-7.

15. Singh AK, Tierney RM, Low DA, et al. A prospective study of differences in duodenum compared to remaining small bowel motion between radiation treatments: implications for radiation dose escalation in carcinoma of the pancreas. Radiat Oncol 2006;1:33.

16. Liu F, Erickson B, Peng C, Li XA. Characterization and management of interfractional anatomic changes for pancreatic cancer radiotherapy. Int J Radiat Oncol Biol Phys 2012;83:e423-9.

17. Sahin B, Zoto Mustafayev T, et al. First 500 fractions delivered with a magnetic resonance-guided radiotherapy system: initial experience. Cureus 2019;11:e6457.

18. Wen N, Kim J, Doemer A, et al. Evaluation of a magnetic resonance guided linear accelerator for stereotactic radiosurgery treatment. Radiother Oncol 2018;127:460-6.

19. El-Bared N, Portelance L, Spieler BO, et al. Dosimetric benefits and practical pitfalls of daily online adaptive MRI-guided stereotactic radiation therapy for pancreatic cancer. Pract Radiat Oncol 2019:9:e46-54

20. Henke L, Kashani R, Robinson $C_{\text {, }}$ et al. Phase I trial of stereotactic MR-guided online adaptive radiation therapy (SMART) for the treatment of oligometastatic or unresectable primary malignancies of the abdomen. Radiother Oncol 2018;126:519-26.

21. Henke L, Kashani R, Yang D, et al. Simulated online adaptive magnetic resonance-guided stereotactic body radiation therapy for the treatment of oligometastatic disease of the abdomen and central thorax: characterization of potential advantages. Int J Radiat Oncol Biol Phys 2016;96:1078-86.

22. Bohoudi 0 , Bruynzeel AME, Senan $S$, et al. Fast and robust online adaptive planning in stereotactic MR-guided adaptive radiation therapy (SMART) for pancreatic cancer. Radiother Oncol 2017; 125:439-44.

23. Wojcieszynski AP, Rosenberg SA, Brower JV, et al. Gadoxetate for direct tumor therapy and tracking with real-time MRI-guided stereotactic body radiation therapy of the liver. Radiother Oncol 2016;118:416-8.

24. Gungor G, Serbez I, Temur B, et al. Time analysis of online adaptive magnetic resonance-guided radiation therapy workflow according to anatomical sites. Pract Radiat Oncol 2021;11:e11-21.

25. Hellman S, Weichselbaum RR. Oligometastases. J Clin Oncol 1995;13:8-10.

26. Palma DA, Olson R, Harrow $S$, et al. Stereotactic ablative radiotherapy versus standard of care palliative treatment in patients with oligometastatic cancers (SABR-COMET): a randomised, phase 2, open-label trial. Lancet 2019;393:2051-8.

27. Rosenberg SA, Henke LE, Shaverdian N, et al. A multi-institutional experience of MR-guided liver stereotactic body radiation therapy. Adv Radiat Oncol 2018;4:142-9.

28. Padgett KR, Simpson G, Asher D, Portelance L, Bossart E, Dogan N. Assessment of online adaptive MR-guided stereotactic body radiotherapy of liver cancers. Phys Med 2020;77:54-63.

29. Barry $A$, McPartlin $A$, Lindsay $P$, et al. Dosimetric analysis of liver toxicity after liver metastasis stereotactic body radiation therapy. Pract Radiat Oncol 2017;7:e331-7.

30. Endrikat JS, Dohanish S, Balzer T, Breuer JA. Safety of gadoxetate disodium: Results from the clinical phase II-III development program and postmarketing surveillance. J Magn Reson Imaging 2015;42:634-43.

31. Jung J, Kim H, Yoon SM, et al. Targeting accuracy of image-guided stereotactic body radiation therapy for hepatocellular carcinoma in real-life clinical practice: in vivo assessment using hepatic parenchymal changes on Gd-EOB-DTPA-enhanced magnetic resonance images. Int J Radiat Oncol Biol Phys 2018;102: 867-74.

32. Jung SH, Yu Jl, Park HC, Lim DH, Han Y. A feasibility study evaluating the relationship between dose and focal liver reaction in stereotactic ablative radiotherapy for liver cancer based on intensity change of Gd-EOB-DTPA-enhanced magnetic resonance images. Radiat Oncol J 2016;34:64-75. 